Rev. Salud pública. 6 (Sup. 1): 50-63, 2004

\title{
Caracterización Clínica y Sociodemográfica de Casos Nuevos de Lepra en Municipios Endémicos y no Endémicos de Colombia
}

\author{
LILIANA CUEVAS ORTIZ ${ }^{1}$, FERNANDO DE LA HOZ², CLARA INÉS LEÓN ${ }^{3}$, \\ MARTHA INÍRIDA GUERRERO ${ }^{4}$ LUIS ARTURO GAMBOA ${ }^{5}$ y MARÍA JULIANA ARAUJO ${ }^{6}$ \\ ${ }^{1}$ Enfermera. M. Sc. Salud Pública. Grupo de Micobacterias. Instituto Nacional de Salud. Bogotá D.C. \\ Colombia. E-mail: eslili@yahoo.com \\ ${ }^{2}$ Médico. Ph. D. Epidemiología. Instituto Colombiano de Ciencia y Tecnología-Colciencias. Bogotá, \\ D.C., Colombia E mail: fdelahoz@colciencias.gov.co. \\ ${ }^{3}$ Bacterióloga. M.Sc Microbiología. Grupo de Micobacterias. Instituto Nacional de Salud. Avenida Calle \\ 26 No 51-60. Bogotá D.C. Colombia. E- mail:_cleon@ins.gov.co \\ ${ }^{4}$ Bacterióloga M. Sc. Microbiologia. Grupo de Micobacterias. Instituto Nacional de Salud. Bogotá, D.C. \\ Colombia. E-mail: mguerrero@ins.gov.co. \\ ${ }^{5}$ Médico Especialista en Dermatología. Centro Dermatológico Federico Lleras Acosta. Bogotá D.C. \\ Colombia \\ ${ }^{6}$ Bacterióloga. Especialista en Epidemiología. Centro Dermatológico Federico Lleras Acosta. Bogotá \\ D.C. Colombia.
}

Recibido 21 Septiembre 2004/Enviado para Modificación 4 Octubre 2004/Aceptado 15 Octubre 2004

\section{RESUMEN}

Objetivo Describir algunas características sociodemográficas, clínicas y epidemiológicas de los casos nuevos de lepra y sus convivientes de nueve municipios endémicos y uno no endémico para lepra en Colombia.

Metodología Se realizó un análisis descriptivo, a partir de la información de los casos nuevos de lepra ingresados al Programa de Control y Eliminación de Lepra de los municipios participantes en el estudio de noviembre de 2002 a octubre de 2003

Resultados El $62,2 \%$ de los casos presentó la forma multibacilar y el $63 \%$ correspondía al género masculino, la mediana de edad fue de 46.5 años. El $55,6 \%$ de los casos nuevos tenía en el momento del diagnóstico discapacidades grado 1 y 2, mayor al reportado a escala nacional (37,5\%) para el año 2003.

Se observó una correlación positiva entre el porcentaje de Necesidades Básicas Insatisfechas por municipio y la incidencia de la enfermedad $(r=0,69$, $\mathrm{p}=0,0180$ ).

Conclusiones Nuestros hallazgos resaltan la poca respuesta del Programa de Control de Lepra en algunos municipios para realizar la detección activa y oportuna de los enfermos, además de evidenciar la asociación que históricamente esta enfermedad ha tenido con la pobreza. 
Palabras Claves: Lepra, endemia, demografía, epidemiología (fuente: Decs BIREME).

|ABSTRACT

Clinical and sociodemographical caracterization of new cases of leprosy in both endemic and non-endemic municipalities in Colombia

Objective To describe some socio-demographical, clinical and epidemiological characteristics of the new cases of leprosy and household reported from 9 endemic and 1 non-endemic towns in Colombia.

Methodology A descriptive analysis was made from the information of the new leprosy cases included in the leprosy control and elimination program of the participant towns in the study carried out between November 2002 and October 2003.

Results $62.2 \%$ of the cases presented the multibacillary form and $63 \%$ of them were males; the median age was 46.5 years. $55.6 \%$ of the new cases had first or second level impairments at the moment of diagnosis. This percentage is higher than the one reported in 2003 at a nationwide level, 37.5 $\%$. A positive correlation was observed between the Unsatisfied Basic Needs Index (NBI) and the disease incidence by town $(r=0.69, p=0.0180)$.

Conclusions Our findings highlight the little response of the Leprosy Control Program in some towns to carry out the active and timely detection of the diseased people, besides making evide

nt the historical relationship that this disease has had with poverty.

Key Words: Leprosy, endemic, demograpy, epidemiology. (Source: MeSh, NLM).

a lepra o enfermedad de Hansen es una enfermedad infecciosa crónica producida por Mycobacterium leprae que afecta preferencialmente el sistema nervioso peri férico, piel, y el tracto respiratorio superior, con especial tropismo por las zonas frías. Esta enfermedad tiene un amplio espectro de presentación dependiendo del tipo de respuesta inmunológica del huésped que va desde el polo tuberculoide al lepromatoso pasando por formas dimorfas. (1) La enfermedad genera serios procesos neurodegenarativos y secundario a esto deformidades importantes cuando no se recibe el tratamiento oportunamente. Además tiene un alto impacto social y psicológico para quienes la padecen debido al estigma que aún se teje sobre ella. (2)

En Colombia existe el Programa de Control y Eliminación de la Lepra (PCL), sus lineamientos técnico-operativos se encuentran en La Guía de Atención de la Lepra (3) definidos por la Dirección General de Promoción y 
Prevención del Ministerio de Salud, y en la ley 715 del 2001 la lepra se incluye como una enfermedad de interés en Salud Pública.

Los objetivos principales del PCL son los de suministrar la terapia multidroga al $100 \%$ de los enfermos, brindarles tratamiento integral e impedir la aparición de discapacidades mediante el diagnóstico oportuno de la enfermedad. El diagnóstico, la notificación y el seguimiento de los enfermos así como el control de los convivientes es responsabilidad de las Empresas Promotoras de Salud (EPS), Administradoras del Régimen Subsidiado (ARS), Instituciones Prestadoras de Salud (IPS) públicas o privadas mediante los planes de beneficio Plan Obligatorio de Salud POS o POS-S, de conformidad con la categoría de afiliación y con cargo a los recursos de subsidio a la oferta para los vinculados. Dentro del Plan de Atención Básica (PAB) se establece como una actividad del PCL la búsqueda activa de casos sospechosos a través del control de convivientes, quienes deben ser canalizados a su respectivo organismo de salud según su afiliación al Sistema General de Seguridad Social en Salud (SGSSS) (3).

Esta enfermedad se ha eliminado en varios países del mundo (4), y en Colombia desde 1997, se logró la meta de eliminación a nivel nacional que es tener menos de 1 caso por 10000 habitantes, pero todavía representa un problema de salud pública para algunos departamentos y municipios en donde esta meta no se ha alcanzado (5).

La eliminación de esta enfermedad a nivel local debe estar soportada en el conocimiento de su distribución y tendencias para redireccionar las estrategias del Programa de Control y Eliminación de Lepra para un control efectivo a través de los organismos estatales, Secretarías de Salud Municipales y Departamentales y organizaciones no gubernamentales que apoyan el control de esta enfermedad. El objetivo del presente estudio fue evaluar algunos aspectos del PCL a través de la descripción de variables clínicas, sociodemográficas, geográficas y epidemiológicas asociadas con enfermedad en los casos nuevos de lepra y sus convivientes en municipios endémicos y no endémicos en el periodo comprendido entre noviembre de 2002 a octubre de 2003.

\section{MATERIALES Y MÉTODOS}

Se realizó un estudio de corte transversal en el cual se incluyeron los casos nuevos de lepra diagnosticados en el periodo comprendido entre noviembre de 2002 a octubre de 2003 en diez municipios endémicos en donde la prevalencia era mayor de 3/10 000 habitantes y uno no endémico con prevalen- 
cia menor a 1/10 000 habitantes, que aceptaron participar en el estudio y firmaron un consentimiento informado.

Se definió como caso de lepra a todo paciente que presentara una o más de las condiciones clínicas de la lepra como son: 1- lesiones cutáneas eritematosas o hipocrómicas con pérdida de la sensibilidad térmica y/o dolorosa o táctil, 2- dolor espontáneo o a la palpación de uno o varios troncos nerviosos periféricos, asociados con signos de afección neural: pérdida sensitiva, disminución de la fuerza muscular o parálisis en el territorio y músculos por ellos inervados, unidas necesariamente a una de las condiciones de laboratorio bacteriológico o histopatológico como son presencia de bacilos ácido alcohol resistentes en frotis de moco y linfa y/o demostración en biopsias de infiltrados inflamatorios que lesionan o destruyen los nervios independiente de la presencia o no de bacilos en la muestra $(3,6)$.

Para recolectar la información de los casos de lepra incluidos en el estudio se diseñó un formulario el cual contenía las variables sociodemográficas como ubicación de la vivienda, tipo de vivienda y acceso a servicios públicos, género y edad entre otras, las variables clínicas como clasificación bacteriológica de la lepra y grado de discapacidad.

Para recolectar la información de los municipios se diseñó otro formulario que contenía las variables de la incidencia y prevalencia de la enfermedad para los últimos cuatro años en cada municipio y la proporción de Necesidades Básicas Insatisfechas (NBI) para el año 2002 (7,8).

La información sobre los casos fue recolectada por el personal de salud encargado del manejo del Programa de Control de Lepra en la Secretaría de Salud de Santander, Secretaria de Salud de Bucaramanga, Sanatorio Agua de Dios, Centro Dermatológico Federico Lleras Acosta y Secretaría Distrital de Salud de Bogotá. Los datos se almacenaron en EPI-Info 6.04.

Durante el mismo período de tiempo ingresaron al estudio los convivientes de los casos nuevos de lepra, a quienes se les descartó la presencia de enfermedad a través del examen clínico. La información de estos convivientes (variables sociodemográficas, tiempo de convivencia con el caso índice, estado de vacunación con BCG y signos clínicos) fue consignada en un formulario diseñado para este fin.

Análisis de la información

Los datos se almacenaron en EPI-Info 6.04. Se calcularon las tasas de prevalencia e incidencia por municipio usando como denominador la población 
reportada por el Departamento Administrativo Nacional de Estadística (DANE) para los municipios en el 2003. Se describieron las características clínicas de los pacientes utilizando porcentajes, promedios o medidas de precisión según el nivel de medición de cada una de las variables.

Se construyo un modelo bivariado de regresión lineal para describir la relación entre NBI e incidencia de lepra. Los estimadores del modelo se obtuvieron usando el método de mínimos cuadrados. Para medir la fuerza de otras asociaciones entre variables discontinuas o discretas, se uso la razón de ventajas ( $\mathrm{RV}$ ó $\mathrm{OR}$ ) con sus $\mathrm{IC}_{95 \%}$. Se realizó un análisis descriptivo mediante análisis univariado y bivariado en EPI 6.04, de los 45 casos que ingresaron en el periodo de estudio.

\section{RESULTADOS}

\section{Características sociodemográficas}

El 63 \% de los casos pertenecían al género masculino, la razón hombre mujer fue 2:1. El rango de edad estuvo entre 21 y 77 años, siendo la mediana de edad 46,5 años, el 44,4 \% de los casos eran menores de 44 años (Tabla 1).

Tabla 1. Distribución de los casos nuevos de lepra del estudio por grupos de edad

\begin{tabular}{ccr}
\hline Grupos de edad & Frecuencia & \multicolumn{1}{c}{$\%$} \\
\hline $15-29$ & 9 & 20,0 \\
$30-44$ & 11 & 24,4 \\
$45-59$ & 16 & 35,6 \\
$60-69$ & 3 & 6,7 \\
70 y más & 5 & 11,1 \\
ND * & 1 & 2,2 \\
TOTAL & 45 & 100,0 \\
\hline * no se obtuvo el dato de edad &
\end{tabular}

El 84,6 \% de los enfermos de lepra provenían del área urbana, el 73,1 \% habitaban en viviendas de ladrillo y un $26,9 \%$ en viviendas de madera y bareque. El 84,6 \% de los casos tenia acceso a servicios de agua y luz, el 61,5 $\%$ a teléfono y a servicio de recolección de basuras el 73,1 \%.

\section{Variables Clínicas}

El 62,2 \% de los casos presentaron la forma multibacilar de los cuales el $67,9 \%$ correspondían a hombres que desarrollaron esta forma (RV:1.88 $\mathrm{IC}_{95 \%} 0,46-7,81 \mathrm{p}=0.32$ ), y dentro del grupo de los paucibacilares la distribución fue muy similar para los dos géneros. La media de edad para los casos 
con forma multibacilar fue de 47,7 años y para los paucibacilares de 42 años $(\mathrm{p}=0,057)$.

Nueve municipios reportaron el $100 \%$ de los casos multibacilares y solo en cinco: Bogotá, Agua de Dios, Bucaramanga, Barrancabermeja y Ocamonte, se reportaron casos paucibacilares

El 55,6 \% de los casos se diagnosticó con grado 1 y 2 de discapacidad. Los multibacilares presentaron un mayor porcentaje de discapacidad grado 1

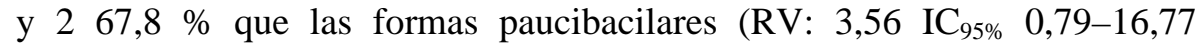
$\mathrm{p}=0,057)$ (Tabla 2).

De los once municipios del estudio solo seis: Bogotá, Agua de Dios, Bucaramanga, Barrancabermeja, Piedecuesta y Molagavita, detectaron menor número de enfermos con discapacidades. En Piedecuesta el $100 \%$ de los casos se detectaron sin discapacidad, seguido de Bogotá con el $87.5 \%$ de los casos sin discapacidad. Los municipios de San Vicente de Chucurí, Socorro, Ocamonte, Molagavita, Floridablanca son los municipios que más aportan casos con discapacidad a pesar de que fueron los municipios que reportaron el menor número de casos durante el periodo de estudio; Barrancabermeja, Agua de Dios y Bucaramanga detectan proporciones similares de enfermos con y sin discapacidad..

Tabla 2. Grado de discapacidad al momento del diagnóstico según forma bacteriológica de la lepra

\begin{tabular}{|c|c|c|c|c|c|c|c|c|c|c|}
\hline \multirow{3}{*}{$\begin{array}{c}\text { Clasificación } \\
\text { OMS }\end{array}$} & \multirow{3}{*}{$N=45$} & \multirow{3}{*}{$\%$} & \multicolumn{8}{|c|}{ Grado de discapacidad al momento del diagnóstico } \\
\hline & & & \multicolumn{2}{|c|}{ Grado 0} & \multicolumn{2}{|c|}{ Grado 1} & \multicolumn{2}{|c|}{ Grado 2} & \multicolumn{2}{|c|}{ ND * } \\
\hline & & & $\mathrm{N}^{\mathrm{O}}$ & $\%$ & $\mathrm{~N}^{\mathrm{O}}$ & $\%$ & $\mathrm{~N}^{0}$ & $\%$ & $\mathrm{~N}^{\mathrm{o}}$ & $\%$ \\
\hline MB & 28 & 62,2 & 8 & 28,6 & 13 & 46,4 & 6 & 21,4 & 1 & 3,6 \\
\hline PB & 17 & 37,8 & 9 & 53 & 3 & 17,6 & 3 & 17,6 & 2 & 11,8 \\
\hline Total & 45 & 100,0 & 17 & & 16 & & 9 & & 3 & \\
\hline
\end{tabular}

* no hay dato; (RV: 3.56 I.C 95\% $0.79-16.77$ p=0.057)

Características de los convivientes de los casos

Se estudiaron 120 convivientes para un promedio de 2,7 convivientes/caso, el tiempo promedio de convivencia con el caso fue de 14 años, el mínimo de 1 año y máximo 60 años. De ellos el 59,3 \% pertenecían al genero femenino, el $35 \%$ eran menores de 15 años y el 73,3 \% convivientes de casos multibacilares. El 75 \% de ellos fueron vacunados con BCG y 9 presentaron algu- 
nos signos clínicos sugestivos de la enfermedad los cuales no fueron suficientes para diagnosticar la lepra.

Descripción epidemiológica de los municipios

La prevalencia reportada en los municipios endémicos en el período de 2000 a 2003 osciló entre 0,2 y 38 casos por 10000 habitantes. La incidencia del 2000 a 2003 osciló entre 1,7 y 131 por 100000 habitantes, es de notar que la mayoría de los municipios no reportó ningún dato de incidencia para 1999. La incidencia y la prevalencia de Bogotá del período 2001-2003, muestran un claro comportamiento de una zona no endémica (Tablas 3 y 4).

Tabla 3. Prevalencia por 10000 habitantes del 2000 - 2003 de los municipios

\begin{tabular}{|c|c|c|c|c|c|c|c|c|}
\hline \multirow{3}{*}{ Municipios } & \multicolumn{8}{|c|}{ del estudio } \\
\hline & \multicolumn{2}{|r|}{2000} & \multicolumn{2}{|c|}{2001} & \multicolumn{2}{|r|}{2002} & \multicolumn{2}{|r|}{2003} \\
\hline & $P^{*}$ & $I C_{95 \%}$ & $P^{*}$ & $\mathrm{IC}_{95 \%}$ & $\mathrm{P}^{*}$ & $\mathrm{IC}_{95 \%}$ & $P^{*}$ & $I_{95 \%}$ \\
\hline Agua de Dios & 37,1 & $27,54-48,78$ & 38,8 & $28,8-50,4$ & 29,3 & $20,64-39,54$ & 22,0 & $15,13-31,86$ \\
\hline Contratación & 22,6 & $12,19-40,47$ & 13,0 & $5,75-28,3$ & 7,5 & $2,4-20,60$ & 5,6 & $1,45-17,9$ \\
\hline Ocamonte & 3,51 & $0,6-14,05$ & 6,93 & $2,23-19,1$ & 5,23 & $1,35-16,65$ & 3,51 & $0,6-14,05$ \\
\hline Piedecuesta & 1,81 & $1,04-2,83$ & 0,42 & $0,13-1,12$ & 0,20 & $0,04-0,82$ & 0,29 & $0,08-0,97$ \\
\hline Molagavita & 1,04 & $0.05-6,63$ & 2,05 & $0,35-8,23$ & 3,06 & $0,79-9,75$ & 5,06 & $1,88-12,65$ \\
\hline $\begin{array}{l}\text { San Vicente } \\
\text { de Chucurí }\end{array}$ & 1,02 & $0,26-3,22$ & 3,0 & $1,25-5,55$ & 2,40 & $1,03-5,1$ & 2,07 & $0,82-4,65$ \\
\hline Floridablanca & 0,86 & $0,52-1.29$ & 0,55 & $0.3-0,94$ & 0,49 & $0,24-0,84$ & 0,24 & $0,1-0,57$ \\
\hline B/bermeja & 0,81 & $0,47-1,32$ & 0,40 & $0,18-0,81$ & 0,45 & $0,22-0,88$ & 0,34 & $0,15-0,75$ \\
\hline Bucaramanga & 0,66 & $0,46-0,91$ & 0,43 & $0,28-0,65$ & 0,40 & $0,25-0,61$ & 0,24 & $0,13-0,42$ \\
\hline Socorro & ${ }^{*} \mathrm{ND}$ & --- & ND & --- & 4,55 & $2,39-8,41$ & 4,55 & $2,39-8,41$ \\
\hline Bogotá & ND & --- & 0,07 & $0,05-0,09$ & 0,07 & $0,05-0,09$ & 0,07 & $0,05-0,09$ \\
\hline
\end{tabular}

Se observó que la incidencia y la prevalencia para algunos de los municipios endémicos siguen siendo bastante alta como Contratación, Agua de Dios, Ocamonte y Molagavita, aún sin tener en cuenta la prevalencia oculta.

En la comparación de la incidencia entre el 2001-2000 y el 2001-2002 para los municipios endémicos, no se observó ningún patrón de tendencia consistente. Sin embargo, se observó un decremento del 50 \% en el 2002 respecto al 2001 en casi todos los municipios a excepción de Barrancabermeja y Contratación donde la incidencia fue igual en estos dos años, mientras que la comparación del 2001 respecto al 2000 mostró un incremento del 
200 \% en San Vicente de Chucurí, del 10 \% en Floridablanca y del 50 \% en Bucaramanga (Figura 1).

Tabla 4. Incidencia por 100000 habitantes del 2000 - 2003 de los municipios del estudio

\begin{tabular}{|c|c|c|c|c|c|c|c|c|}
\hline \multirow[t]{2}{*}{ Municipio } & \multicolumn{2}{|c|}{2000} & \multicolumn{2}{|r|}{2001} & \multicolumn{2}{|c|}{2002} & \multicolumn{2}{|c|}{2003} \\
\hline & I* & $\mathrm{IC}_{95 \%}$ & 1 * & $\mathrm{IC}_{95 \%}$ & I* & $\mathrm{IC}_{95 \%}$ & I* & $\mathrm{IC}_{95 \%}$ \\
\hline Contratación & 131 & $57-284$ & 56 & 14. 6- 180 & 56 & $14.6-180$ & 56 & $14.6-180$ \\
\hline Agua de Dios & 94.9 & $52-166$ & 65 & 32.1-129.6 & 29.2 & $9.4-80.3$ & 29.2 & $9.4-80.3$ \\
\hline Ocamonte & 34.8 & $6-140$ & 17.4 & $0.9-113.1$ & 34.8 & $6-140.5$ & 0.0 & 0 \\
\hline Molagavita & 20.4 & $3.5-82.3$ & ND & --- & 40.8 & $13.1-122$ & 10.2 & $0.5-66.3$ \\
\hline Piedecuesta & 7.1 & $3.1-15.4$ & 4.0 & $1.3-11.2$ & 2.0 & $0.4-8.2$ & 1.0 & $0.1-6.6$ \\
\hline $\begin{array}{l}\text { San Vicente } \\
\text { de Chucurí }\end{array}$ & 6.7 & $1.2-27.2$ & 20.2 & $8.2-46.5$ & 10.1 & $2.6-32.2$ & 10.1 & $0.2-32.2$ \\
\hline Floridablanca & 3.2 & $1.5-6.8$ & 3.6 & $1.8-7.3$ & 2.4 & $1.0-5.7$ & 2.1 & $1.0-4.8$ \\
\hline $\mathrm{B} /$ manga & 2.5 & $1.5-4.5$ & 3.7 & $2.3-5.8$ & 2.7 & $1.6-4.7$ & 2.0 & $1.1-3.8$ \\
\hline $\mathrm{B} /$ bermeja & 1.9 & $0.6-5.4$ & 1.9 & $0.6-5.4$ & 1.9 & $0.6-5.4$ & 1.9 & $0.6-5.4$ \\
\hline Socorro & *ND & --- & ND & --- & ND & --- & 4.1 & $0.2-26.9$ \\
\hline Bogotá & ND & --- & 0.5 & $0.3-0.75$ & 0.3 & $0.2-0.54$ & 0.4 & $0.3-0.65$ \\
\hline
\end{tabular}

I *: Incidencia; ND: sin dato para estos años

Figura 1. Incremento - Decremento de la incidencia de lepra 2000-2002

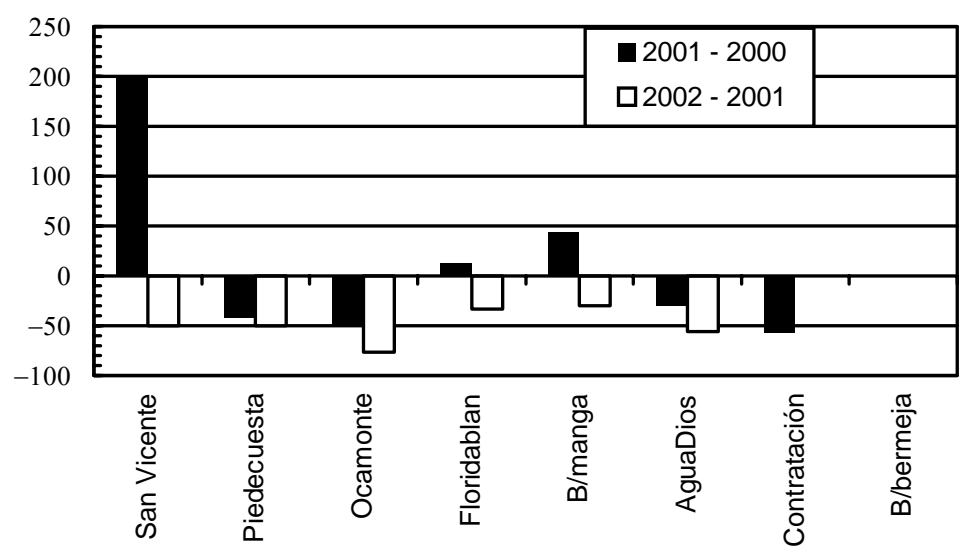

El modelo lineal detectó una correlación positiva con el porcentaje de NBI por municipio (5) y la proporción de casos nuevos para el año $2002\left(\mathrm{r}^{2}\right.$ : 0,4809 r: 0,6934 p=0,018) (Figura 2). 


\section{DISCUSIÓN}

Pese al carácter descriptivo del estudio se logró identificar algunos hallazgos que pueden contribuir a mejorar el programa de Control. En primer lugar algunos de nuestros resultados sugieren que la meta de eliminación aún no se cumple para algunos municipios del país. Una de estas evidencias es que hay una gran proporción de casos entre 15 y 44 años siendo muy inferior esta proporción en los grupos de mayor edad. Si la enfermedad estuviera al borde de la eliminación se presentaría lo contrario, como lo muestran estudios realizados en Noruega y Portugal donde esta enfermedad se eliminó completamente a principios del siglo XX y comienzos de la década de los 80 del siglo pasado respectivamente $(9,10)$.

Debido a que el periodo de incubación de la lepra es largo, llegando a ser para las formas multibacilares hasta de 20 años, (11) la presencia de la enfermedad entre los pacientes jóvenes sugiere que la infección se esta adquiriendo a temprana edad.

Adicionalmente, muchos de los convivientes de los enfermos de lepra son menores de 15 años (12) y para ellos se haría necesario un seguimiento más cercano y estricto que lo recomendado por la Guía de Atención de Lepra ya que la exposición al bacilo de lepra a edades mas tempranas puede asociarse con una mayor probabilidad de enfermarse (13).

Se sugiere una vigilancia que debe mantenerse durante un periodo mínimo de 5 años, una vez que el paciente ha completado el tratamiento, la vigilancia de los convivientes de un caso paucibacilar debería mantenerse durante un periodo mínimo de 2 años una vez terminado el tratamiento, si no es posible los convivientes deberían ser examinados al menos en una ocasión durante este periodo. (6)

Otra evidencia de que la meta de eliminación está aún lejana es el hecho de que la mayoría de los casos diagnosticados son multibacilares lo cual coincide con lo reportado por el resto de los municipios colombianos con lepra $68,5 \%$ de casos son multibacilares. (14)

Por otro lado, nuestro estudio evidencia diferencias en la organización del programa de control entre los diferentes municipios. En solo cinco de los once municipios se reporta la forma paucibacilar, demostrando que allí existe una infraestructura adecuada y personal capacitado para el diagnóstico y tratamiento de los enfermos de lepra como seria el caso del Sanatorio de 
Agua de Dios y el Instituto Dermatológico Federico Lleras Acosta en Bogotá.

Figura 2. Comparación del Porcentaje de NBI y tasa de incidencia por 100000 habitantes por municipio. 2002

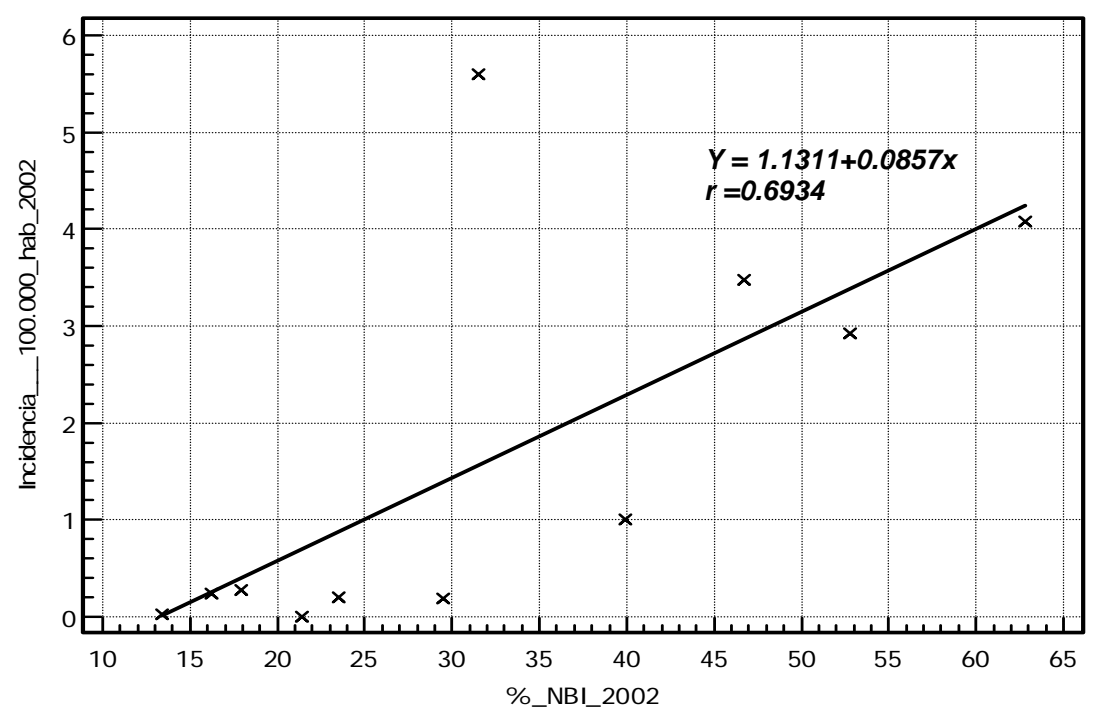

Es necesario que las directivas nacionales del programa evalúen la capacidad operativa de control de la lepra en los seis municipios restantes donde no se presentan las formas paucibacilares, ya que este hallazgo sugiere probablemente una debilidad en los procedimientos de diagnóstico dado que las formas paucibacilares son mas difíciles de diagnosticar. La dificultad podría radicar en que en estos municipios, podrían estar haciendo el diagnóstico únicamente con base en la baciloscopia, lo cual es inadecuado ya que este es un diagnóstico esencialmente clínico que debe ser complementado con la biopsia de piel o nervio, la baciloscopia se usa para clasificar la enfermedad y establecer el tratamiento $(3,6)$. Análisis de años anteriores han mostrado un alto número de recaídas en los casos paucibacilares (5), lo cual refuerza la sospecha de una clasificación inadecuada de los casos de lepra y por tanto un esquema inadecuado de tratamiento.

En lo referente a la detección de discapacidades en el momento del diagnostico: el grado de discapacidad 1 se relacionó en una mayor proporción con la forma multibacilar, observación que difiere de lo que comúnmente se 
esperaría ya que son las formas paucibacilares las que generan mayor discapacidad por la afección precoz de los troncos nerviosos (1).

Esta distribución se podría explicar por la detección tardía de los casos, ya que se sabe que deben haber transcurrido mínimo dos años después de la aparición de los primeros signos y síntomas para observar discapacidades (15). Llama la atención que en los municipios en donde se ubican los centros especializados para el diagnóstico y tratamiento de los enfermos, es donde se reportan las formas paucibacilares y menor proporción de enfermos con discapacidades.

Analizando la forma como opera el Programa de Control y Eliminación de Lepra en las dos instituciones especializadas del área endémica se observa que en Agua de Dios la cantidad de personal para atender la población del área aunque no llega a ser de 1 por 1000 habitantes están dedicados tiempo completo a las actividades del PCL lo cual se traduce en una mayor efectividad de las acciones del PCL. Igualmente en Contratación el personal tiene una dedicación de tiempo completo y realizan actividades de búsqueda activa.

En el resto de los municipios endémicos, el PCL se desarrolla en los organismos estatales de salud especialmente de primer nivel, a excepción de San Vicente de Chucurí en donde existe un hospital de segundo nivel pero con escaso personal dedicado al PCL, esto puede ser una de las causas de que todos sus pacientes son detectados tardíamente con discapacidad 1 y 2 . Ocamonte es el municipio que más personal tiene por 1000 habitantes , cuenta con varios promotores de salud responsables de las actividades del PCL, a pesar de lo cual detecta el 100\% de los casos con discapacidad 1 y 2. $\mathrm{Al}$ parecer la deficiencia esta en que las actividades están delegadas a los promotores de salud y auxiliares de enfermería quienes se encargan de realizar únicamente charlas a la comunidad. En Floridablanca no se llevan a cabo las actividades del PCL como responsabilidad del municipio si no que las actividades las realiza el personal de salud de Bucaramanga, por lo tanto allí no hay apropiación de las actividades y responsabilidad del PCL, encontrándose que el $100 \%$ de los casos son detectados con discapacidad 1 y 2.

Todo lo anterior podría estar determinado que en los municipios en donde se desarrollan permanentemente las actividades del programa sean los que mantienen tasas de incidencia más altas que aquellos en donde no, por lo cual en los municipios con menores tasas de incidencia y prevalencia no estaría más cercana la eliminación, si no que se estaría reflejando la escasa búsqueda de casos. 
En conclusión el alto porcentaje de discapacidad grado 1 y 2 detectado en el momento del diagnostico (55,6 \%), evidencia claramente la baja capacidad de respuesta del Programa de Control y Eliminación de Lepra, especialmente en los municipios que detectan el $100 \%$ de sus casos con discapacidad, porcentaje que esta muy por encima de lo reportado a nivel nacional que para el 2003 fue del 37,5 \% para discapacidad grado 1 y 2 (14). La discapacidad es otro elemento que indicaría que la meta de eliminación seguiría siendo aplazada, ya que las personas detectadas tardíamente con discapacidades han sido focos de diseminación de la enfermedad por largo tiempo al no recibir tratamiento oportuno.

A pesar de que en el análisis individual de algunas características socioeconómicas como el acceso a servicios públicos es optimo, el análisis ecológico entre la proporción de personas con NBI por municipio y la incidencia de lepra muestra que hay una correlación positiva entre un mayor porcentaje de NBI (6) y mayores tasas de incidencia, lo cual corrobora que la lepra es una enfermedad que en nuestro país sigue ligada a la pobreza, lo que puede seguir constituyendo una gran barrera para que se avance a una verdadera eliminación de la lepra a nivel subnacional. (2-16-17).

Dentro de lo que le compete al sector de la salud, se propone que se fortalezcan los servicios de primer nivel de atención, responsabilidad transferida a los municipios a partir del decreto 77 de 1987 y que se continuó normando en los siguientes años con las Leyes 10/90, 60/93 y 100/93 (18).

La Atención Primaria en Salud $(19,20)$ se debe retomar no como las acciones de menor costo, es decir aquello que solo puedan realizar los promotores de salud, sino como las actividades esenciales pero óptimas e integrales dirigidas al individuo y a los colectivos con las cuales se garantice la detección oportuna y el correcto manejo de las personas que padecen de lepra, enfermedad en la cual su diagnóstico es realmente sencillo y no tiene porque manejarse adecuadamente solo en centros de segundo y tercer nivel de atención. Para esto los municipios deben conocer y monitorizar su verdadera situación epidemiológica respecto a la lepra y establecer metas a mediano y corto plazo con lo cual se generen estrategias operativas sostenidas como la búsqueda de casos (21) para la eliminación de esta enfermedad (22, 23). También sería importante identificar y exponer cuales son los problemas estructurales y coyunturales que repercuten en el escaso impacto del Programa de Control y Eliminación de Lepra en el ámbito local mediante un estudio de caso (24) en donde se aborden los procesos de sociopolíticos y técnicos de dicho programa en el nivel municipal y departamental. 
Se concluye que la lepra es una enfermedad que en algunos municipios realmente no esta en etapa de eliminación, que existe un subregistro de casos lo que establecería una prevalencia oculta la cual debe estimarse para establecer metas de búsqueda activa y así mismo optimizar los recursos del PCL, también se deben retomar estrategias como el seguimiento de los convivientes teniendo en cuenta el alto riesgo de estas personas de desarrollar la enfermedad.

Agradecimientos: Este estudio se desarrollo gracias a la financiación de Colciencias (Proyecto código 2104-04-11798, CT 133-2002) y del Instituto Nacional de Salud de Colombia. Los autores agradecen a los enfermos y a sus convivientes por su colaboración y participación en el estudio. También al personal de salud encargado de la coordinación del Programa de Control de Lepra de los municipios que participaron en el estudio: Martha Stella Sarmiento, Enfermera y Angélica Ribera, Bacterióloga Sanatorio de Agua de Dios, Colombia; Lilia Edith López. Enfermera. Secretaria Distrital de Salud Bogotá, Colombia; Esteban César Numa. Médico, Sergio Córdoba, Técnico Programa Lepra, Isabel Osorio de Mosquera, Enfermera, Liliana Chaparro, Enfermera, Secretaria de Salud de Santander, Colombia; José Antonio Rodríguez, Médico, Secretaria Local de Salud Barrancabermeja, Colombia; Dary Cardona, Enfermera. Secretaria Local de Salud de Molagavita, Colombia; Socorro Fajardo, Enfermera, Secretaria Local de Salud de Piedecuesta, Colombia; Gladys Acero, Enfermera, Secretaria Local de Salud de Socorro Hospital San Juan de Dios, Socorro, Colombia; Sonia Janeth Caballero, Enfermera, Secretaria Local de Salud de San Vicente de Chucurí, Hospital San Juan de Dios, Colombia

\section{REFERENCIAS}

1. Rodríguez G, Orozco LC. Lepra. Santa Fe de Bogotá D.C: Instituto Nacional de alud; 1996.

2. Frazão L, Leuba M. A reprodução social da Hanseníase: um estudo do perfil de doentes com hanseníase no Município de Sao Pãolo Cad. Saúde Pública, Rio de Janeiro 2002;18:101-113.

3. Ministerio de Salud Dirección General de Promoción y Prevención. Guía de Atención de la Lepra; 2002.

4. Lockwood D. Leprosy elimination a virtual phenomenon or a reality. BMJ 2002;234:12-6.

5. Guerrero MI, Plazas N, León CI. Situación de la lepra en Colombia: un análisis crítico. Biomédica 2000;20:266-271.

6. OMS. Una guía para el control de la lepra. $2^{\text {a }}$ ed. Madrid: Ministerio de Sanidad y Consumo: 1988.

7. Secretaria de Salud de Santander. Necesidades Básicas Insatisfechas Municipios de Santander hallado en: 
http://www.camaradirecta.com/webccb/temas/salud/cifras/nbi.html. Acceso 24 marzo 2004.

8. DANE-DAPD. Encuesta calidad de vida 2003. Distribución de personas por NBI compuesto total hallado en: http//www.dapd.gov.co/www/section-1877jsp. Acceso 24 marzo 2004.

9. Meima A, Irgens L, Gerrit J, Habberma D. Disappearance of leprosy from Norway an exploration of critical factors using epidemiological modelling aproach. Int J Epidemiol 2002;31:991-1000.

10. Irgens I, Melo C, Lechat M. Leprosy in Portugal 1946-80: epidemiologic patterns observed during declining, incidence rates. Lepr Rev 1990;6:3249.

11. Fine P. Leprosy: The Epidemiology of a slow bacterium. Epidemiol Rev 1982;4:161-184.

12. Ministerio de Salud. INS. Situación de Salud en Colombia Indicadores Básicos 2002.

13. Jains S, Reddy R, Osmani S, Lockwood D, Sunnetha S. Childhood leprosy in a urban clinic. Hyderabab, India: clinical presentation and the role of household contacts. Lepr Rev 2002;73:248-53.

14. Instituto Nacional de Salud, Ministerio de la Protección Social. Consolidado de casos y actividades del Programa Nacional de Eliminación de Lepra. 2003.

15. Meima A, Saunderson P, Gebre S, Desta K, Oortmarssen G, Habbema D. Factors associated with impairments in mew leprosy patients: the AMFES cohort. Lepr Rev 1999;70:189-203.

16. Mackeown T. Los orígenes de las enfermedades humanas. Barcelona: Editora Crítica; 1990.

17. Mani M. Ecological factors in transmission of leprosy. Indian $\mathrm{J}$ Lepr 1996;68:375-7.

18. Jaramillo I. La descentralización del sector salud en Colombia. Balance del proceso de descentralización. Bogotá, Ministerio del Interior.1997.

19. OMS/UNICEF. Atención primaria de salud, Informe de la Conferencia Internacional sobre Atención Primaria de Salud, Alma-Ata (URSS), Sept. 6-12 de 1978. Ginebra: OMS/UNICEF.

20. OPS. La atención primaria en salud,viejos y nuevos retos. Disponible en:http//www.cor.ops - oms.org/texto completo/configured redirect.Acceso mayo 2004.

21. Tiendrebeago A, Sow O, Traora M, Sissoko K, Coullbaly B. Comparison of two methods of leprosy finding in the circle of Kita in Mali. Int J Epidemiol 1999;67:237-242.

22. WHO. Remaining challenges towards the elimination of leprosy. Leprosy Elimination Project. World Health Organization. Indian J Lepr 2000;72:3345I.

23. Durrheim, Speare R. Global leprosy elimination: time to change more than the elimination target date. J Epidemiol Community Health. 2003;57:316-317.

24. Silva LR, Herrera V, Agudelo CA. Promoción, prevención, municipalización y aseguramiento en salud, en siete municipios. Rev Salud Pública. 2002; 4:36-58. 